\title{
Low Cost Binaural System Based on the Echolocation
}

\author{
Thiago Fernandes Moya Moreira ${ }^{1(\otimes)}(\mathbb{D})$ José Lima $^{2}\left(\mathbb{D}\right.$, Paulo Costa $^{3}(\mathbb{D}$, \\ and Márcio Cunha ${ }^{1} \mathbb{D}$ \\ 1 Academic Department of Electronic, Federal University of Technology - Paraná, \\ Curitiba, Brazil \\ thiagomoya@ymail.com \\ 2 INESC TEC - INESC Technology and Science and CeDRI - \\ Research Centre in Digitalization and Intelligent Robotics, \\ Polytechnic Institute of Bragança, Bragança, Portugal \\ 3 INESC TEC - INESC Technology and Science \\ and Faculty of Engineering of University of Porto, Porto, Portugal
}

\begin{abstract}
Ultrasonic sensors offers attractive features at an affordable cost. The main problem faced by the use of these devices is that the data obtained are not so easy to interpret, restricting their efficiency. This paper describes a binaural sensor system that is able to determine the coordinates of an object or a target in a two-dimensional space, focusing on mathematical and signal processing techniques to provide accurate measurements and increase the system reliability. The proposed work consists only of low cost components, which aims to demonstrate that improvement is possible. Experimental tests, performed in different scenarios, reported good accuracy and repeatability of the measurements.
\end{abstract}

Keywords: Ultrasonic sensor $\cdot$ Echolocation $\cdot$ Object detection

\section{Introduction}

In mobile robot applications the knowledge of the environment in which the device is inserted and the objects that surround it are essential information for a successful navigation, avoiding collisions and increasing the system safety. To achieve this goal the use of sensors becomes indispensable and its features must be taken into account [1]. Among the different types of sensors, many researches have been exploring the ultrasonic sensors to deal with this problem attracted by the low cost, flexibility in several environments and applications where vision based sensors fail due to some constraints like low visibility, mirrors or clear objects [2].

Typically, the employment of ultrasonic sensing in an industrial or commercial atmosphere is restricted to simple tasks, such as obstacles avoidance that do not require accurate measurements. The leading reason for this scenario is due to the fact that the data obtained from the commercial ultrasonic range-finders are 
not easy to be interpreted. Therefore, the literature has shown a great interest in investigating the use of these sensors for complex applications [3-5].

The result of a measurement performed by a standard sensor occurs through an ultrasonic burst with a single frequency, which can range from $40 \mathrm{kHz}$ to 50 $\mathrm{kHz}$. The distance is calculated using the Time of Flight (ToF) when the echo emitted from the transmitter reflects an obstacle or object and returns back to the receiver [6]. In general, the reflected signal is detected by a predefined trigger level, however this method is not so efficient [7]. Thus, to improve the system accuracy, some enhanced approaches have combined advanced signal processing techniques $[8,9]$.

Conventional ultrasonic range-finders mostly are composed of a single transmitter and a single receiver or a transducer that acts as both. The main disadvantage of this system is the wide beam of the emitted signal, causing a very poor resolution. Furthermore, other phenomena like the multi-reflection effect may arise and cause false positives. In order to reduce these issues, a system that contains at least two receivers and a transmitter is needed [6].

This paper proposes a low cost ultrasonic binaural system that combines several mathematical and signal processing techniques to improve the accuracy and performance of the system. Also, the characteristics associated with the use of ultrasonic measurements in indoor environments are discussed.

After this brief introduction, in Sect. 2, a review about ultrasonic sensing is performed. In Sect. 3, necessary techniques to develop the system are presented. In Sect. 4, the architecture of the created system is introduced. In Sect. 5, the main objective of the proposed work and the procedures to reach it are described. In Sect.6, the results of the experimental tests are reported. Lastly, in Sect. 7 the results are discussed and conclusions drawn.

\section{Ultrasonic Sensing}

The operating principle used by acoustic sensors, such as ultrasonic, is mainly based on the ToF estimation. The transmitter generates an ultrasonic pulse which propagates through the air and when it detects a target the signal is reflected back to the receiver. In this sense, the ToF can be determined by the time the signal is emitted and received by the sensor [10]. Equation 1 shows how the distance measurement is achieved from the estimated ToF.

$$
d=\frac{c \cdot T_{f}}{2}
$$

where $c$ is the sound velocity and $T_{f}$ is the ToF.

As previously mentioned, most of the conventional ultrasonic ranging systems uses a value as reference to establish the exact point when the signal reflected is detected by the receiver. As long as the echo amplitude exceeds the predefined threshold level, there will always be a reading, which occurs at the time $t_{0}[11]$, as shown in Fig. 1.

Threshold detection does not require complex calculations and can be easily implemented, but the ToF estimation is not so reliable. The problem arises from 


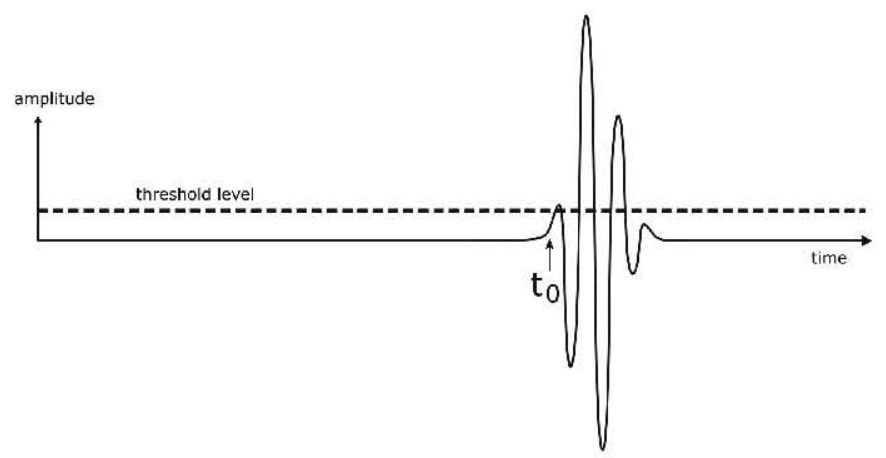

Fig. 1. Typical echo of the ultrasonic ranging system [11].

the fact that a signal will always have a rising time, so this condition added to other factors compromises the efficiency of this approach [12].

\section{System Requirements}

In this Section are introduced the fundamental concepts and techniques for the ultrasonic binaural system development, associating optimized approaches to enhance the system performance.

\subsection{ToF Estimation Methods}

In Sect. 2 the major drawback involving the threshold detection technique is addressed. Thus, to handle with this situation some alternatives used in this work are presented in the following subsections.

\subsubsection{Digital Envelope Detection}

Envelope detection is accomplished using analog or digital resources. Analog methods works well if it is done carefully. Nevertheless, the best performing analog circuits are complex and delicate. In addition, according to [13] analog processing is not suitable to the envelope detection of digital data.

In the digital methods, approaches that extract the analytic signal magnitude are explored. For this purpose the Hilbert transform is commonly used to generate an analytic signal from a real signal, whose absolute value represents the envelope of the echo signal received [14]. This technique offers great precision, however it has a high computational consumption, involves complex calculus and in certain cases this method may be unstable [13]. For this reason a different approach is adopted.

In [15] a novel method, i.e. cubic spline interpolation method to extract the envelope from a signal is presented. Interpolation is one of the most important methods of numerical approximation, which allows to establish new data points from a set of known data points. To perform the envelope extraction, a windowing is applied to the signal to locate the peaks, and after that, the unknown 
points between each peak are interpolated. Unlike the first one, the procedures to execute this method does not require complex operations. A second order Butterworth low pass filter is designed to soften the envelope. Figure 2a and b shows an example of a received echo signal and the envelope before and after the filter, respectively. The phase delay caused by the filter is negligible. Finally, from the extracted envelope the ToF is determined by the maximum value of the signal.

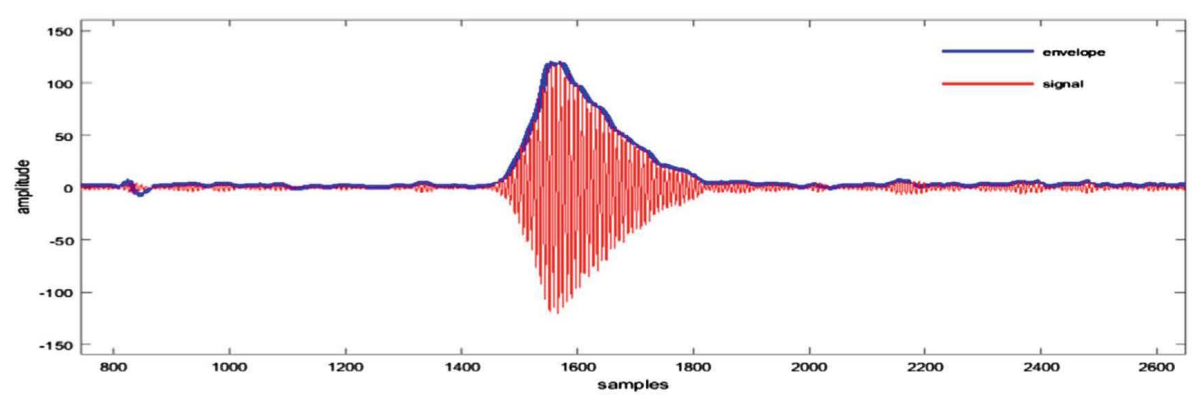

(a) Raw envelope.

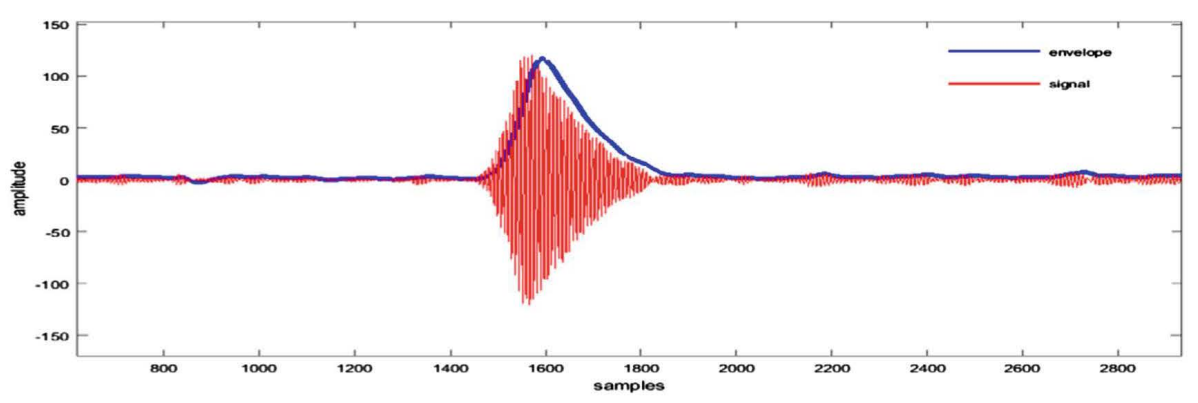

(b) Filtered envelope.

Fig. 2. Extracted envelope from the echo signal.

\subsubsection{Cross-Correlation}

Cross-correlation (CCR) is an important method to determine the time delay between two signals. When this method is applied, it produces a peak at the time delay and a reduction in the noise level. Usually the CCR takes a transmitted and received signal and yields a new signal in the time domain which the maximum value happens at the delay time [8]. Equation 2 demonstrates how the CCR can be calculated.

$$
c(t)=\int_{-\infty}^{+\infty} s_{T}(t) s_{R}(t+\tau) d(\tau)
$$


where $s_{T}(t)$ is the transmitted signal and $s_{R}(t+\tau)$ is the signal received and shifted in time.

The transmitted signal by the sensor generates eight cycle sonic burst at $40 \mathrm{kHz}$, i.e. it provides eight square pulses with period of $25 \mu \mathrm{s}$. Then, in this case, applying CCR between the transmitted and received signal would not be appropriate. To implement this idea it would be necessary to detect the transmitted signal in a waveform similar to that of the received signal, as can be seen in Fig. 2. For this reason, the CCR is applied only to the received signals from sensor A and B (Fig. 6). Knowing the time lag it is possible to establish which of the receiving sensors is closest to the target.

\subsection{Triangulation}

Triangulation is a procedure to determine the position of an object or a target from known points using trigonometric techniques. In general, the number of dimensions of the estimated position of an object is directly related to the number of sensors, for instance, two sensors can indicate a location in 2D, while three sensors can indicate a 3D location and so on [16].

In geometry, when the length of the sides of any triangle is known a theorem to calculate its area can be applied. This approach was created by the mathematician Heron of Alexandria and has been used in many mathematical applications [17]. With the distance of the baseline and the distance calculated by each sensor known, there is the possibility to estimate the area of the triangle formed (Fig. 3) using Heron's formula, as shown in Eq. 3.

$$
\begin{gathered}
\Delta(a b c)=\sqrt{p(p-a)(p-b)(p-c)}, \\
\text { where } p=\frac{a+b+c}{2}
\end{gathered}
$$

whose $a, b, c$ are the known sides. Alternatively, the area of a triangle can be computed using the Eq. 5 .

$$
\Delta(a b c)=\frac{b \cdot h}{2}
$$

where $b$ is the base and $h$ is the height of the triangle. Reorganizing Eq. 5 is possible obtain Eq. 6 .

$$
h=\frac{\Delta(a b c) \cdot 2}{b}
$$

If the area provided by Eq. 3 is placed in Eq. 6 the y-coordinate of the target can be determined. By separating the original triangle into two right triangles and after that applying the Pythagorean Theorem it is possible to calculate the x-coordinate, according to Eqs. 7 and 8.

$$
\begin{aligned}
& C_{1}=\sqrt{d_{A}^{2}-h^{2}} \\
& C_{2}=\sqrt{d_{B}^{2}-h^{2}}
\end{aligned}
$$




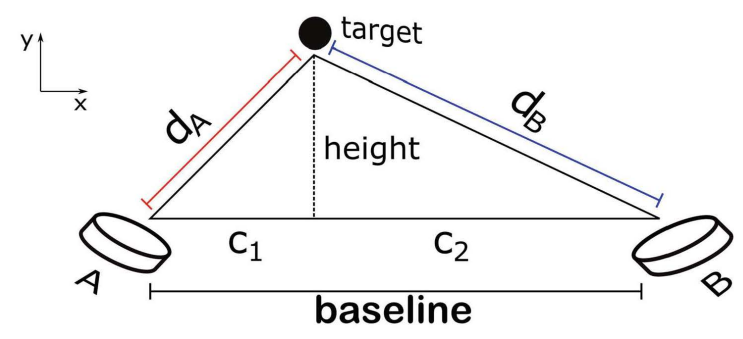

Fig. 3. Triangulation created by the sensors.

where $h, C_{1}, C_{2}, d_{A}$ and $d_{B}$ are shown in Fig. 3. If the Eq. 7 is used, the system reference for the coordinates on the $\mathrm{x}$-axis will originate from sensor $\mathrm{A}$, otherwise, from sensor B.

Valid calculations but nonsense physical can arise using the equations described above. Thus, in order to avoid incoherent measurements some restrictions are established. A typical error is shown in Fig. 4, to avoid it, it is observed that $\theta_{1}$ and $\theta_{2}$ angles are outside of the detection area.

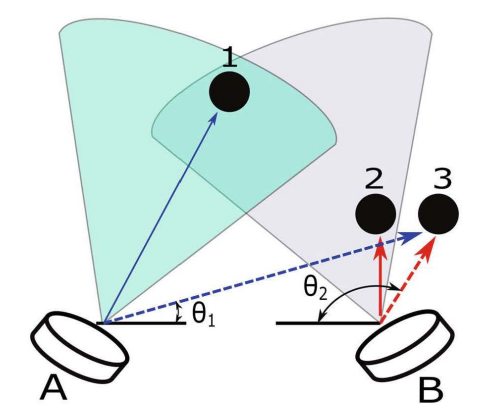

Fig. 4. Typical triangulation error. Adapted from [16].

Figure 4 shows that sensor A detects the target 1 and sensor B detects the target 2 . Nonetheless, $\theta_{1}$ and $\theta_{2}$ are not valid, which causes an error resulting in a false positioning, represented by the target 3 .

For a valid detection area Fig. 5 displays how $\theta_{1}$ and $\theta_{2}$ should be, indicating that $\theta_{1}$ has to be equal $\alpha_{1} \pm \phi$, where $\alpha_{n}$ is the angular position of the sensor $n$ corresponding to the $\mathrm{x}$-axis and $\phi$ is half the angle of the detection beam, which this value is particular to each sensor model. Then, the angular interval can be given by $\cos \left(\alpha_{1}-\phi\right) \leq \cos \theta_{1} \leq \cos \left(\alpha_{1}+\phi\right)$. Similarly, to sensor $\mathrm{B}$ the angular limiting range is $\cos \left(\alpha_{2}-\phi\right) \leq \cos \theta_{2} \leq \cos \left(\alpha_{2}+\phi\right)$, where

$$
\cos \theta_{1}=\frac{l^{2}+d_{A}^{2}-d_{B}^{2}}{2 l d_{A}}
$$

and

$$
\cos \theta_{2}=\frac{l^{2}+d_{B}^{2}-d_{A}^{2}}{2 l d_{B}}
$$


$\alpha_{1}, \alpha_{2}$ and $\phi$ are constant, then, it is necessary to calculate only $\cos \theta_{1}$ and $\cos \theta_{2}$. Measurements outside this range are discarded.

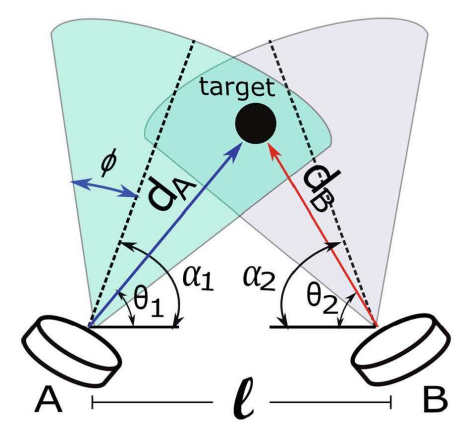

Fig. 5. Example of a valid detection. Adapted from [16].

\section{System Architecture}

The system consists in three low-cost ultrasonic sensors HY-SRF05 and a microcontroller (MCU) STM32F103C8T ARM Cortex-M3 with two built-in analog to digital converters. The ultrasonic burst is achieved by sensor $\mathrm{C}$, and the raw echo signal received by sensors $\mathrm{A}$ and $\mathrm{B}$ is sent to the 12 bits A/D converters of the MCU with a sampling rate of $320 \mathrm{kHz}$.

Adopting the speed of sound as $340 \mathrm{~m} / \mathrm{s}$, it can be stated that the distance is covered $0.034 \mathrm{~cm} / \mu \mathrm{s}$. The mutual is equal to $29.412 \mu \mathrm{s} / \mathrm{cm}$, and considering a round trip corresponds to $58.824 \mu \mathrm{s} / \mathrm{cm}$. Using this value it is possible to simplify the determination of the distance and substitute the Eq. 1 by the Eq. 11 .

$$
d=\frac{T_{f}}{K}
$$

where $K \approx 59$.

The measurements from each sensor are stored in an array with four thousand positions, which if multiplied by the sampling period, $T s=3.125 \mu \mathrm{s}$, corresponds to a sample space of $12500 \mu \mathrm{s}$. Using the Eq. 11 we can estimate that the proposed system has a maximum range of about $212 \mathrm{~cm}$ depth, that is, along the y-axis. The $\mathrm{x}$-axis is limited by the established baseline.

After obtaining the data, the samples are transmitted over RS-232 protocol to a computer to be processed by the MATLAB software. Finally, the calculation of the object coordinates is accomplished.

\subsection{Binaural Sonar System}

Binaural sonar system is composed of two receivers and one emitter, as shown in Fig. 6. This arrangement aims to collect more information and increase the 


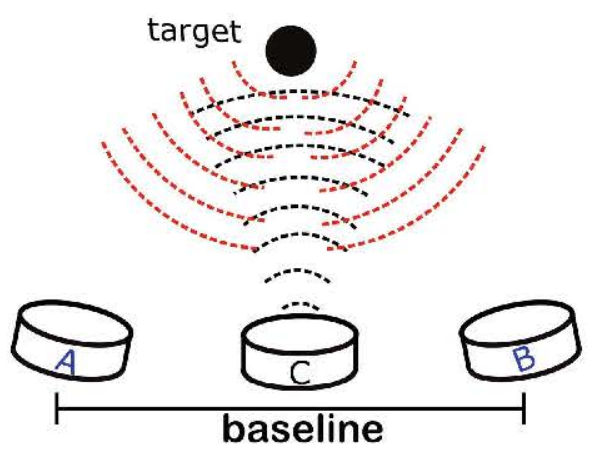

Fig. 6. The binaural sensor system.

accuracy of the measurements. Also, according to [6] a multi-sonar system is able to reduce significantly the problems faced by the use of a single sensor.

The designed system has three conventional ultrasonic sensors, which two acts as receivers and one as emitter. The region overlapped by the sensors, as seen in Fig. 7, is called detection area and any object contained in that space is able to be located by the system. The receivers, sensors A and B, are separated by a known baseline $(l)$ which through the application of trigonometric techniques allows to calculate the spatial coordinates of an object in a two-dimensional plane. Adjusting the angle and baseline, there is the possibility of changing the detection area when the two ultrasonic beam patterns overlap.

The best positioning is defined according to the baseline and the $\alpha$ angle from experimental tests.

\section{Proposed Work}

The major objective of the proposed work is to improve the performance of the binaural system joining the methods presented in Subsect.3.1, to define with high accuracy the $\mathrm{x}$ and $\mathrm{y}$ axis coordinates of one or more targets existing in the detection area through the triangulation.

As mentioned in Sect.3.1.2, the CCR is performed only with the received signals. From the known time delay between the sensors A and B is possible to determine which of the sensors receives the echo first and consequently if it is closer or not to the object. In spite of obtaining this information, it is not enough to estimate the ToF, because it is necessary to have some reference of the origin, i.e. when the signal is transmitted.

In order to solve the issue previously quoted, the technique described in Sect. 3.1.1 is employed to find out the ToF of one of the received echoes. So, the digital envelope detection is applied to the sensor closest to the target, believing that accuracy may be better. Following, the ToF of the other sensor is defined based on the time delay provided by the CCR. For example, suppose the sensor $A$ is the closest sensor to the target and the delay time given by CCR is $2 \mathrm{~ms}$ advanced from sensor $\mathrm{B}$. Then, the ToF of the sensor $\mathrm{B}$ will be the ToF of 
the sensor A subtracted $2 \mathrm{~ms}$. If sensor B were the closest sensor, the opposite analogy would be used.

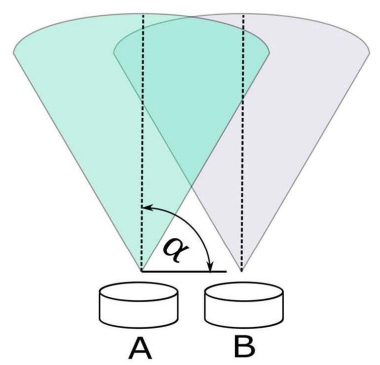

Fig. 7. Detection area formed by the ultrasonic beam patterns. Adapted from [16].

\section{Results}

The main purpose of the experimental tests is verify the accuracy of the measurements and analyze the general performance of the proposed system. The tests are divided into two stages, the first with only one object and the second with two objects present in the detection area. In the former, a small box is used and the receiver sensors are placed with $\theta_{1,2}=45^{\circ}$ and $l=51 \mathrm{~cm}$. The experiments are performed with the object positioned to the left, right and center of the detection area. About sixty samples of each possibility are obtained and the standard deviation $(\sigma)$, mean $(\overline{(x, y)})$ and relative error of each one are calculated, as can be seen in Table 1.

Table 1. Mean, standard deviation and relative error of the first tests.

\begin{tabular}{l|l|l|l|l|l}
\hline Location & $(x, y)$ real $(\mathrm{cm})$ & $\overline{(x, y)}$ estimated $(\mathrm{cm})$ & $\sigma_{x}(\mathrm{~cm})$ & $\sigma_{y}(\mathrm{~cm})$ & Relative error (\%) \\
\hline Left & $(11.5,91.8)$ & $(11.14,91.57)$ & 1.41 & 0.36 & 0.30 \\
\hline Center & $(25.6,84.5)$ & $(24.42,80.45)$ & 0.01 & 0.08 & 4.78 \\
\hline Right & $(35.0,86.4)$ & $(35.98,84.59)$ & 1.01 & 0.86 & 1.39 \\
\hline
\end{tabular}

Since the raw echo signal is used, there is the possibility to do a wide investigation about the measured environment. If a second object is present in the detection field, this information can be discovered through the existence of more than one crest in the waveform of the echo signal. In the second stage, the object described before is used together with a larger box, and the same procedures are applied. The positioning of both objects is arbitrary, the smaller box is closer to the sensors and the larger box further away. The standard deviation $(\sigma)$, mean $(\overline{(x, y)})$ and relative error of each situation are presented in Table 2. 
Table 2. Mean, standard deviation and relative error of the further tests.

\begin{tabular}{l|l|l|l|l|l}
\hline Box & $(x, y)$ real $(\mathrm{cm})$ & $\overline{(x, y)}$ estimated $(\mathrm{cm})$ & $\sigma_{x}(\mathrm{~cm})$ & $\sigma_{y}(\mathrm{~cm})$ & Relative error $(\%)$ \\
\hline 1 & $(35.5,54.0)$ & $(33.04,53.52)$ & 0.39 & 0.27 & 2.67 \\
\hline 2 & $(19.0,111.2)$ & $(19.58,110.29)$ & 1.09 & 0.21 & 0.71 \\
\hline
\end{tabular}

When the CCR is applied to the received echoes a signal with triangular shape is produced, resulting in an improper time delay. Probably this situation occurs because both signals has a significant direct current (DC) offset, then, to deal with this problem, the offset removal is performed through the normalization of the data, subtracting each sample by the arithmetic mean of all samples.

Particularly in the second stage, to use CCR when more than one object is in the scene, it is necessary to separate the echo signals received by the sensors according to the crests of the waveform, as can be seen in Fig. 9, and use the CCR in each of them with its corresponding crest. For instance, the CCR would have to be applied to the first part of the sensor A with the first part of the sensor B, and so on. Otherwise, if the entire signal is used, a single time delay is provided and consequently is not possible to distinguish which target that delay is referring to.

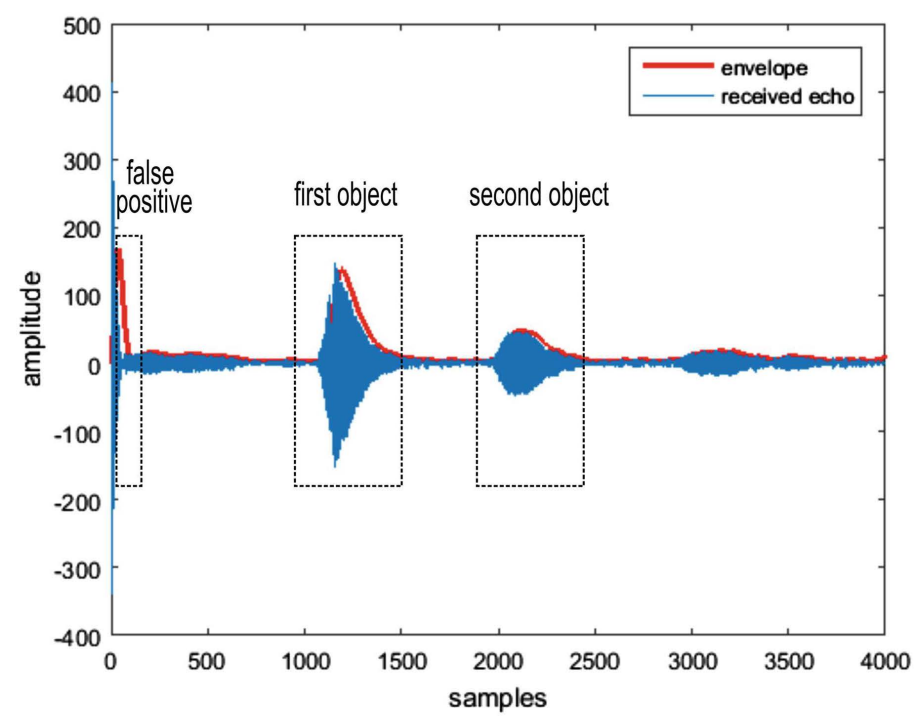

Fig. 8. Received echo signal with two objects in the detection area.

Figure 8 shows a frequent error in the receiver sensors. The object is more than $1 \mathrm{~cm}$ apart, however the system indicates that the object is at a smaller distance. The problem seems to occur because the echo signal interferes with a signal that travels directly from the transmitter to the receiver, without reflecting on the object. Thus, in an attempt to reduce incoherent data the first samples are ignored. 


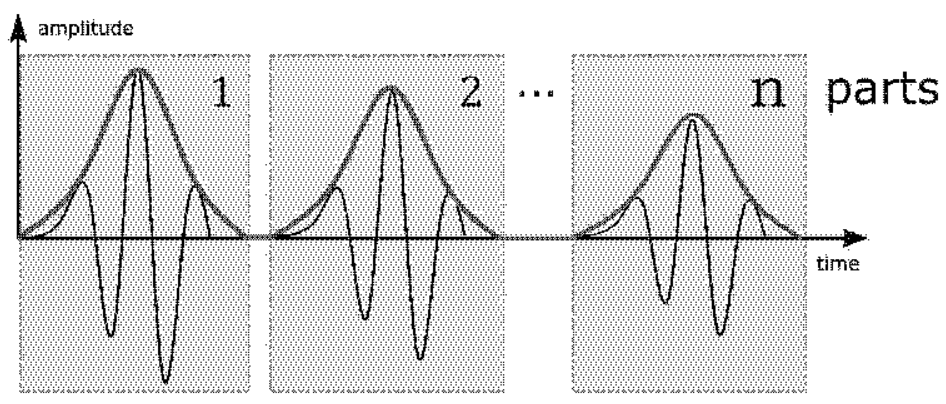

Fig. 9. Fragmentation of the received echo signal.

\section{Conclusion and Future Work}

A low cost binaural sensor system was described in this paper, that was able to detect the coordinates of one or more objects present in the detection area and provide a deeper analysis of the environment. Triangulation and signal processing techniques were used to improve the system performance. Experimental tests confirmed the accuracy of the measurements from the obtained results, which the mean system error for the first scenario with a single object, and for the second with two objects was $2.16 \%$ and $1.69 \%$ respectively.

As future work, it is intended to optimize the processing time with another $\mathrm{MCU}$, classify the shape of the detected object and embed all to some mobile navigation device for testing and system validation. In general, the binaural sensor system using mathematical and signal processing techniques can be an attractive alternative to the conventional models of ultrasonic sensing, presenting good accuracy and repeatability of the measurements.

Acknowledgement. This work is financed by the ERDF-European Regional Development Fund through the Operational Programme for Competitiveness and Internationalisation-COMPETE 2020 Programme within project POCI-01-0145FEDER-006961, and by National Funds through the FCT—Fundação para a Ciência e a Tecnologia (Portuguese Foundation for Science and Technology) as part of project UID/EEA/50014/2013.

\section{References}

1. Lim, Z.S., Kwon, S.T., Joo, M.G.: Multi-object identification for mobile robot using ultrasonic sensors. Int. J. Control Autom. Syst. 10(3), 589-593 (2012)

2. Walter, C., Schweinzer, H.: Locating of objects with discontinuities, boundaries and intersections using a compact ultrasonic 3D sensor. In: 2014 International Conference on Indoor Positioning and Indoor Navigation (IPIN), pp. 591-600. IEEE (2014)

3. Bank, D.: A novel ultrasonic sensing system for autonomous mobile systems. IEEE Sens. J. 2(6), 597-606 (2002)

4. Cong-Nguyen, H., Oh, S., Kim, N., Park, D., Han, S.: Real-time robust control of mobile robot using ultrasonic sensor. In: 2008 International Conference on Control, Automation and Systems, pp. 2585-2589. IEEE (2008) 
5. Jawale, R.V., Kadam, M.V., Gaikawad, R.S., Kondaka, L.S.: Ultrasonic navigation based blind aid for the visually impaired. In: 2017 IEEE International Conference on Power, Control, Signals and Instrumentation Engineering (ICPCSI), pp. 923928. IEEE (2017)

6. Kreczmer, B.: Objects localization and differentiation using ultrasonic sensors. In: Robot Localization and Map Building. IntechOpen (2010)

7. Kaniak, G., Schweinzer, H.: A 3D airborne ultrasound sensor for high-precision location data estimation and conjunction. In: 2008 IEEE Instrumentation and Measurement Technology Conference, pp. 842-847. IEEE (2008)

8. Jackson, J.C., Summan, R., Dobie, G.I., Whiteley, S.M., Pierce, S.G., Hayward, G.: Time-of-flight measurement techniques for airborne ultrasonic ranging. IEEE Trans. Ultrason. Ferroelectr. Freq. Control 60(2), 343-355 (2013)

9. Khyam, M.O., Ge, S.S., Li, X., Pickering, M.R.: Highly accurate time-of-flight measurement technique based on phase-correlation for ultrasonic ranging. IEEE Sens. J. 17(2), 434-443 (2016)

10. Andria, G., Attivissimo, F., Giaquinto, N.: Digital signal processing techniques for accurate ultrasonic sensor measurement. Measurement 30(2), 105-114 (2001)

11. Kuc, R., Siegel, M.W.: Physically based simulation model for acoustic sensor robot navigation. IEEE Trans. Pattern Anal. Mach. Intell. PAMI-9(6), 766-778 (1987)

12. Queirós, R., Martins, R.C., Girão, P.S., Serra, A.C.: A new method for high resolution ultrasonic ranging in air. In: Proceedings International Measurement Confederation, Rio de Janeiro (2006)

13. Fritsch, C., Ibanez, A., Parrilla, M.: A digital envelope detection filter for real-time operation. IEEE Trans. Instrum. Measur. 48(6), 1287-1293 (1999)

14. Xu, B., Yu, L., Giurgiutiu, V.: Advanced methods for time-of-flight estimation with application to Lamb wave structural health monitoring. In: Proceedings International Workshop on SHM, pp. 1202-1209 (2009)

15. Jia, X.F., An, H.Q., Zhang, S.G.: Cubic spline interpolation method for the envelope tracking of middle and low frequency voltage flicker. In: Advanced Materials Research, pp. 704-709. Trans Tech Publications (2014)

16. Gearhart, C., Herold, A., Self, B., Birdsong, C., Slivovsky, L.: Use of ultrasonic sensors in the development of an Electronic Travel Aid. In: 2009 IEEE Sensors Applications Symposium, pp. 275-280. IEEE (2009)

17. Bényi, Á.: 87.47 a heron-type formula for the triangle. Math. Gaz. 87(509), 324-326 (2003) 